\title{
Semantic Web for Lîfe Sciences: vision, aims, tools, platforms
}

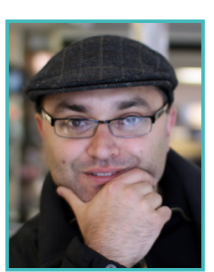

Andrea Splendiani is an independent professional (intelliLeaf Itd) and adjunct lecturer at DERI.

He earned a Laurea Degree in Information Technology from the Politecnico di Milano, and a $\mathrm{PhD}$ in Computer Science from the University of Milano-Bicocca. He has been working in functional genomics for immunology (Genopolis) and Systems Biology (Institut Pasteur) where he has been an active contributor to the BioPAX standard. He has then worked on biomedical ontologies (University of Rennes 1) and data integration (Rothamsted Research, BBSRC).

$\mathrm{He}$ is co-chair and organizer of SWAT4LS (Semantic Web Applications and Tools for Life Sciences) and Guest Editor of the Journal of Biomedical Semantics.

$\mathrm{He}$ is currently collaborating with different companies on data modeling for healthcare and life sciences, as well as semantic solutions for knowledge and data management.

This tutorial is an introduction to Semantic Web technologies for Bioinformaticians, Researchers and other professionals working on biomedical and life sciences data. It is composed of two parts: an introduction to technologies and tools, and an overview of how these technologies fit in the current bioinformatics landscape.

The first part will briefly introduce key concepts and technologies (RDF, OWL, Rules, SKOS, SPARQL, Linked-Data, Triplestores) and then present tools and techniques to address two prototypical use cases: publishing information on the Semantic Web, and consuming information that can be found in RDF.

The second part of the tutorial will present an overview of the state of adoption of Semantic Web technologies in bioinformatics (which databases publish information in RDF and different representation patterns). It will also present features and limitations that characterize these technologies, to provide an understanding of which data and applications they can practically benefit.

Perspective participants are invited to get in contact with the presenter so that the content of the tutorial can be tuned to their specific interests. See his Linkedln page. 